\title{
Group Theoretical Symmetries and Generalized Bäcklund Transformations for Integrable Systems
}

\author{
Guido Haak \\ Institut für Theoretische Physik, FU-Berlin \\ Arnimallee 13, D-1000 Berlin 33
}

\begin{abstract}
We present a notion of symmetry for 1+1-dimensional integrable systems which is consistent with their group theoretic description and reproduces in special cases the known Bäcklund transformation for the generalized Korteweg-deVries hierarchies. We also apply it to the relativistic invariance of the Leznov-Saveliev systems.
\end{abstract}

In [1] a general and detailed group theoretic description of integrable systems, based on the work of Segal, Wilson, Reyman and Semenov-Tian-Shansky was given. It included the work of Drinfel'd and Sokolov on the generalized modified Korteweg-deVries (mKdV) hierarchy and the notion of a generalized Miura transformation for these systems. The aim of this work is to interpret some of the well known properties of integrable systems in this group theoretic setting.

As the relation between the Bäcklund transformation, Miura transformations and symmetries of differential equations was one of the hallmarks of the development of the inverse scattering method (for an account on this see e.g. [2]), it is of interest to exhibit the relationship between these structures and the group theoretic approach. Thus an aim will be to establish an appropriate notion of a symmetry and in particular to explain the Bäcklund transformation of the $\mathrm{KdV}$ models for the group $\mathrm{SL}(n, \mathbb{C})$ in terms of the group theoretic setting. 
The first section gives a short description of the group theoretic formulation as presented in [四]. The examples of the generalized nonlinear Schrödinger (NLS) equation and the sine-Gordon equations are then presented in more detail.

In the second section we present the formulation of a symmetry and relate it by the generalized Miura transformation of Drinfel'd, Shabat and Sokolov [3, 4] to the Bäcklund transformation in close analogy to the well known case of the KdV hierarchy. We give an explicit reduction for the group theoretic hierarchy of the generalized NLS equation, which produces the $\mathrm{mKdV}$ flows, and a Bäcklund transformation for the generalizations of the $\mathrm{KdV}$ equation belonging to the groups $\mathrm{SL}(n, \mathbb{C})$.

At the end we show, how this kind of symmetry can be generalized to include the Lorentz group symmetry of the Leznov-Saveliev systems.

\section{The Group Theoretical Setting}

Following [1] an integrable system is given in terms of the following abstract data:

a) An admissible triple of Lie-Groups $\left(\mathbf{G}, \mathbf{G}_{-}, \mathbf{G}_{+}\right)$with $\mathbf{G}_{ \pm} \subset \mathbf{G}$ closed, the multiplication map $\mathbf{G}_{-} \times \mathbf{G}_{+} \rightarrow \mathcal{U}$ being a diffeomorphism into an open dense subset $\mathcal{U}$ of $\mathbf{G}$. This is an abstract Birkhoff factorization. In addition the following requirements are made:

1) The corresponding Lie algebras form a Manin triple $\left(\mathbf{g}, \mathbf{g}_{-}, \mathbf{g}_{+}\right)$, i.e. there exists a Killing form $B_{G}$ on $\mathbf{g}=\mathbf{g}_{-} \oplus \mathbf{g}_{+}$such that $\mathbf{g}_{-}$ and $\mathbf{g}_{+}$are maximally isotropic w.r.t. $B_{G}$.

2) The exponential map $\exp : \mathbf{g}_{-} \rightarrow \mathbf{G}_{-}$is bijective.

b) An element $p \in \mathbf{g}_{+}$is fixed.

The Birkhoff factorization [5] is clearly modeled to generalize the decomposition of $\mathrm{GL}(n, \mathbb{C})$ into upper and lower triangular matrices to infinite dimensional groups. Note also that $\mathbf{g}_{+}$(and $\mathbf{g}_{-}$) bears the additional structure of a Lie Bialgebra [6] induced by the Manin triple $\left(\mathbf{g}, \mathbf{g}_{-}, \mathbf{g}_{+}\right)$.

$p$, called the generalized momentum, will play the rôle of the generator of space translations. The $x$-space flow $g_{-}(x)$ in $\mathbf{G}_{-}$for small $x \in \mathbb{R}$ through an 
element $g_{-}=g_{-}(0) \in \mathbf{G}_{-}$is defined in the group theoretically most natural way via

$$
g(x)=\exp (-x p) g_{-}=g_{-}(x) g_{+}(x),
$$

where the second equation denotes the Birkhoff factorization of $g(x)$.

In the following we choose a Cartan subalgebra $\mathbf{h}$ of $\mathbf{g}$, such that $p$ is an element of $\mathbf{h}_{+}=\mathbf{h} \cap \mathbf{g}_{+}$. We may then take any element of $\mathbf{h}_{+}$to generate further local flows in the group $\mathbf{G}$, which commute with the $x$-flow. We write them in the compact form

$$
g(\underline{\mathrm{t}})=\exp (-\langle\underline{\mathrm{t}}, \underline{\mathrm{p}}\rangle) g_{-},
$$

where $\underline{\mathrm{t}}=\left(t_{0}=x, t_{1}, \ldots\right), \underline{\mathrm{p}}=\left(p_{0}=p, p_{1}, \ldots\right)$. It is always understood that the parameters $t_{j} \in \mathbb{R}$ are small, and only finitely many are nonzero.

In the forthcoming examples $\mathbf{G}$ is a loop group with infinite rank. Therefore the systems described above possess infinitely many commuting flows, giving also an infinite set of integrals of motion.

The connection with the realization of integrable systems as zero curvature conditions (Zakharov-Shabat (ZS) type equations) is now given by choosing the natural flat connection on the trivial principal bundle $\mathbf{G} / \mathbf{G}_{+} \supset \mathbf{G}_{-}$, the pullback of the (right invariant) Maurer-Cartan form on $\mathbf{G}_{+}$with the map $g_{+}: \mathbf{G} \rightarrow \mathbf{G}_{+}$:

$$
\omega=-\mathrm{d} g_{+} g_{+}^{-1}
$$

The Maurer-Cartan equation,

$$
\mathrm{d} \omega+[\omega, \omega]=0,
$$

translates into a zero curvature condition of the ZS type for the components $V_{j}(\underline{\mathrm{t}})$ of the connection in the direction of the right invariant vector fields $r\left(p_{j}\right)$ for $p_{j}$ :

$$
\begin{gathered}
V_{j}(\underline{\mathrm{t}})=\omega\left(r\left(p_{j}\right)\right)(g(\underline{\mathrm{t}}))=q_{+}\left(\operatorname{Ad}\left(g_{-}^{-1}(\underline{\mathrm{t}})\right) p_{j}\right), \\
\frac{\mathrm{d} V_{k}(\underline{\mathrm{t}})}{\mathrm{d} t_{j}}-\frac{\mathrm{d} V_{j}(\underline{\mathrm{t}})}{\mathrm{d} t_{k}}-\left[V_{k}(\underline{\mathrm{t}}), V_{j}(\underline{\mathrm{t}})\right]=0,
\end{gathered}
$$

together with an auxiliary equation

$$
g_{-}^{-1}(\underline{\mathrm{t}}) \frac{\mathrm{d} g_{-}(\underline{\mathrm{t}})}{\mathrm{d} t_{j}}=-q_{-}\left(\operatorname{Ad}\left(g_{-}^{-1}(\underline{\mathrm{t}})\right) p_{j}\right) .
$$


Here we denote by $q_{ \pm}$the projections $\mathbf{g} \rightarrow \mathbf{g}_{ \pm} \cdot U(\underline{\mathrm{t}})=V_{0}(\underline{\mathrm{t}})$ is by (5) and the fact that $\mathbf{g}_{-}$is maximally isotropic w.r.t. a nondegenerate form $B_{G}$, a generic element of the orbit of the coadjoint action of $G_{-}$on $\mathbf{g}_{+}$through $p$. The Lie-Kirillov-Kostant-Souriau symplectic structure on this orbit gives a Poisson structure being compatible with the one given by the Lie Bialgebra $\mathrm{g}_{+}$.

The equation (5) gives a map $\Omega_{p_{0}}$ from the coset $\mathbf{H}_{-}\left(p_{0}\right) \backslash \mathbf{G}_{-}, \mathbf{H}_{-}\left(p_{0}\right)$ being the centralizer of $p_{0}$ in $\mathbf{G}_{-}$, to the set $\mathcal{K}_{0}\left(\mathbb{R}, \mathbf{g}_{+}\right)$of smooth functions defined in a neighborhood of the origin in $\mathbb{R}$ with values in $\mathbf{g}_{+}$. We assume this map to be injective. For a fixed admissible triple $\left(\mathbf{G}, \mathbf{G}_{-}, \mathbf{G}_{+}\right)$this is a restriction on the momentum operator $p_{0}$.

Restricting attention to the case of solutions $U(\underline{\mathrm{t}})$ periodic in $x$ (say with period $l$ ), we may use the assumed injectivity of $\Omega_{p_{0}}$ to define for every periodic solution $U(x)$ a periodic element $\tilde{g}_{-}(x)$ of $\mathbf{G}_{-}$by

$$
\tilde{g}_{-}(x)=\exp (-x I) g_{-}(x),
$$

where $g_{-}(x)$ is any $x$-space flow in $\mathbf{G}_{-}$mapped by $\Omega_{p_{0}}$ to $U(x)$ and $I \in \mathbf{h}_{-}$ is defined by

$$
g_{-}(l)=\exp (l I) g_{-}(0) .
$$

Notice that $g_{-}(x)$ and $\tilde{g}_{-}(x)$ are in the same equivalence class modulo $\mathbf{H}_{-}\left(p_{0}\right)$.

Combining equations (5) and (7) for $j=0$ this gives

$$
\begin{aligned}
\omega\left(r\left(p_{0}\right)\right)(g(x)) & =\operatorname{Ad}\left(g_{-}^{-1}(x)\right) p_{0}+g_{-}^{-1}(x) \frac{\mathrm{d} g_{-}(x)}{\mathrm{d} x} \\
& =\operatorname{Ad}\left(\tilde{g}_{-}^{-1}(x)\right)\left(p_{0}+I\right)+\tilde{g}_{-}^{-1}(x) \frac{\mathrm{d} \tilde{g}_{-}(x)}{\mathrm{d} x} .
\end{aligned}
$$

Using Frenkel's definition [7] of the affine Lie group $\mathcal{L} \mathbf{G}$ connected with $\mathbf{G}$ this may be rewritten as

$$
\omega\left(r\left(p_{0}\right)\right)(g(\cdot))+1 \mathcal{D}=\widetilde{\operatorname{Ad}}\left(\tilde{g}_{-}(\cdot)\right)\left(p_{0}+I+I_{0} \mathcal{C}+1 \mathcal{D}\right)
$$

where $I_{0}=-l B_{G}\left(p_{0}, I\right)$.

The periodic $x$-space flows may thus be incorporated into the group theoretic scheme by substituting the admissible triple $\left(\mathbf{G}, \mathbf{G}_{-}, \mathbf{G}_{+}\right)$by a triple $\left(\mathcal{L} \mathrm{G}, \mathcal{L} \mathrm{G}_{-}, \mathcal{L} \mathrm{G}_{+}\right)$which is also admissible [1, Lemma 2.6]. 
As an example let us take the triple of analytic loop groups

$$
\left(\mathbf{G}, \mathbf{G}_{-}, \mathbf{G}_{+}\right)=\left(L^{a} \mathbf{K}_{\mathbb{q}}, L_{1}^{a-} \mathbf{K}_{\mathbb{C}}, L^{a+} \mathbf{K}_{\mathbb{C}}\right),
$$

$\mathbf{K}$ a compact semisimple Lie group, $\mathbf{K}_{\mathbb{C}}$ its complexification with Lie algebra $\mathbf{k}_{\mathbb{q}}$.

In order to achieve bijectivity of the exponential map exp : $\mathbf{g}_{-} \rightarrow \mathbf{G}_{-}$, we don't use the usual construction of a Loop group over $\mathbf{K}_{\mathbb{C}}$ but the one given in [1]. There the group $L^{a} \mathbf{K}_{\mathbb{q}}$ is defined to be the stalk at infinity of the sheaf of analytic functions on $\mathbb{C}$, i.e. $L^{a} \mathbf{K}_{\mathbb{C}}$ consists of functions which are defined and analytic outside some compact set. The subgroup $L^{a+} \mathbf{K}_{\mathbb{C}}$ consists of the entire maps from $\mathbb{C}$ to $\mathbf{K}_{\mathfrak{C}}$, and $L_{1}^{a-} \mathbf{K}_{\mathfrak{d}}$ consists of the maps, which are defined and analytic in some open neighborhood of $\infty$ and have the value 1 there. Their Lie algebras are $\left(L^{a} \mathbf{k}_{\mathbb{C}}, L_{0}^{a-} \mathbf{k}_{\mathbb{C}}, L^{a+} \mathbf{k}_{\mathbb{C}}\right)$ with self-explanatory notation. The choice of the Killing form

$$
B_{L^{a} \mathbf{K}_{\mathbb{q}}}(a(\cdot), b(\cdot))=\operatorname{Res}_{\lambda=\infty}\left(B_{\mathbf{K}_{\mathbb{T}}}(a(\lambda), b(\lambda))\right)
$$

makes this triple of Lie algebras into a Manin triple.

For $p_{0}=\lambda A, A \in \mathbf{h}_{\mathbb{C}}$ with $\mathbf{h}_{\mathbb{G}}$ being the Cartan subalgebra of $\mathbf{k}_{\mathbb{T}}$, the resulting integrable system is the NLS hierarchy, generalizing the vector-NLS of Fordy and Kulish [ [8]. By a reduction described in the next section we get the mKdV type systems.

For all these systems and all generic $p_{0}$, injectivity of $\Omega_{p_{0}}$ holds and the integrals of motion can be calculated explicitly, as can be easily seen by generalizing the proof in [1] for $p_{0}=\lambda A$ to arbitrary $p_{0}$.

A second important example is the sine-Gordon equation and its generalizations, the Leznov-Saveliev systems [9], in light-cone variables.

They belong to the following construction, also considered in [10]. Given a Birkhoff factorization $\left(\mathbf{G}, \mathbf{G}_{-}, \mathbf{G}_{+}\right)$as before, we may construct another factorization by setting:

$$
\widetilde{\mathbf{G}}=\mathbf{G} \times \mathbf{G}, \widetilde{\mathbf{G}}_{-}=\mathbf{G}_{-} \times \mathbf{G}_{+}, \widetilde{\mathbf{G}}_{+}=\{(g, g) \in \widetilde{\mathbf{G}}\} \cong \mathbf{G} .
$$

The splitting of an element $\left(g_{1}, g_{2}\right) \in \widetilde{\mathbf{G}}$ is given by the Birkhoff factorization: If $g_{1} g_{2}^{-1}=g_{-} g_{+}^{-1}, \hat{g}=g_{-}^{-1} g_{1}=g_{+}^{-1} g_{2}$, then

$$
\left(g_{1}, g_{2}\right)=\left(g_{-}, g_{+}\right)(\hat{g}, \hat{g}) .
$$


On the Lie algebra level this reads:

$$
\widetilde{\mathbf{g}}=\mathbf{g} \oplus \mathbf{g}, \widetilde{\mathbf{g}}_{-}=\mathbf{g}_{-} \oplus \mathbf{g}_{+}, \widetilde{\mathbf{g}}_{+}=\operatorname{diag}(\widetilde{\mathbf{g}})
$$

With the Killing form $B_{\widetilde{G}}\left(\left(x_{1}, x_{2}\right),\left(y_{1}, y_{2}\right)\right)=B_{G}\left(x_{1}, y_{1}\right)-B_{G}\left(x_{2}, y_{2}\right)$ this becomes a Manin triple.

If we restrict the $p_{j}$ to $\mathbf{g}_{+} \oplus \mathbf{g}_{+}$then the orbit and all flows are restricted to this subset, which is isomorphic to $\mathbf{g}_{+}$, and the equations (5) and (7) are the same as for the Birkhoff factorization $\left(\mathbf{G}, \mathbf{G}_{-}, \mathbf{G}_{+}\right)$. This is the reason why the flows of the mKdV systems commute with the flows of the LeznovSaveliev ones as mentioned in [4].

However, to recognize the higher symmetry of these systems, connected with their Lorentz invariance, it is more convenient to use Drinfel'd's double construction:

Given a Lie group $\mathbf{G}$ with Lie algebra $\mathbf{g}$ being a Kac-Moody algebra, $\mathbf{h}$ a Cartan subalgebra and $\mathbf{n}_{ \pm}$the standard nilpotent subalgebras (see below), we denote by $\mathbf{N}_{ \pm}$and $\mathbf{H}$ the subgroups of $\mathbf{G}$ belonging to them. We set:

$$
\begin{gathered}
\mathcal{D} \mathbf{G}=\mathbf{G} \times \mathbf{G}, \\
\mathcal{D} \mathbf{G}_{+}=\{(g, g) \in \mathcal{D} \mathbf{G}\} \cong \mathbf{G}, \\
\mathcal{D} \mathbf{G}_{-}=\left\{\left(n_{-} h, n_{+} h^{-1}\right) \in \mathcal{D} \mathbf{G} \mid n_{ \pm} \in \mathbf{N}_{ \pm}, h \in \mathbf{H}\right\} .
\end{gathered}
$$

The factorization of an element $\tilde{g}=\left(g_{1}, g_{2}\right) \in \mathcal{D} \mathbf{G}$ is achieved by using the Gauss decomposition $g_{1} g_{2}^{-1}=n_{-} h^{2} n_{+}^{-1}$ : If $\hat{g}=h^{-1} n_{-}^{-1} g_{1}=h n_{+}^{-1} g_{2}$, then

$$
\tilde{g}=\left(g_{1}, g_{2}\right)=\left(n_{-} h, n_{+} h^{-1}\right)(\hat{g}, \hat{g}) .
$$

On the Lie algebraic level this is the classical double $\mathcal{D} g$. Given an element $(x, y) \in \mathcal{D}$ g, we may write

$$
(x, y)=\left(x_{-}+\frac{1}{2} x_{\mathbf{h}}, x_{+}-\frac{1}{2} x_{\mathbf{h}}\right)+(\hat{x}, \hat{x}),
$$

with $x_{-}-x_{+}+x_{\mathbf{h}}$ being the linear decomposition of $x-y$ into an $\mathbf{n}_{-}, \mathbf{n}_{+}$ and $\mathbf{h}$ part, and $\hat{x}=x-x_{-}-\frac{1}{2} x_{\mathbf{h}}=y-x_{+}+\frac{1}{2} x_{\mathbf{h}}$.

Here, if $p_{0}$ is contained in one of the Lie subalgebras $\mathbf{n}_{ \pm}$(identify $\widetilde{\mathbf{g}}_{+}$with g), we have no injectivity of $\Omega_{p_{0}}$ (see also [10]). This can be seen directly 
by looking at the expressions for $U(\cdot)=\omega\left(r\left(p_{0}\right)\right)(\tilde{g}(\cdot))$ : Let the splitting of $\tilde{g} \in \widetilde{\mathbf{G}}$ be as above and abbreviate $n_{-} h, n_{+} h^{-1}$ by $g_{-}, g_{+}$respectively, then

$$
\omega\left(r\left(p_{0}\right)\right)(\tilde{g}(\underline{\mathrm{t}}))=q_{+}\left(\operatorname{Ad}\left(g_{-}^{-1}(\underline{\mathrm{t}})\right) p_{0}\right)+q_{-}\left(\operatorname{Ad}\left(g_{+}^{-1}(\underline{\mathrm{t}})\right) p_{0}\right),
$$

and the auxiliary equation splits into:

$$
g_{-}^{-1}(\underline{\mathrm{t}}) \frac{\mathrm{d} g_{-}(\underline{\mathrm{t}})}{\mathrm{d} x}=-q_{-}\left(\operatorname{Ad}\left(g_{-}^{-1}(\underline{\mathrm{t}})\right) p_{0}\right)+q_{-}\left(\operatorname{Ad}\left(g_{+}^{-1}(\underline{\mathrm{t}})\right) p_{0}\right)
$$

and

$$
g_{+}^{-1}(\underline{\mathrm{t}}) \frac{\mathrm{d} g_{+}(\underline{\mathrm{t}})}{\mathrm{d} x}=-q_{+}\left(\operatorname{Ad}\left(g_{+}^{-1}(\underline{\mathrm{t}})\right) p_{0}\right)+q_{+}\left(\operatorname{Ad}\left(g_{-}^{-1}(\underline{\mathrm{t}})\right) p_{0}\right) .
$$

$q_{+}$and $q_{-}$being the projection given by (21), i.e. $q_{ \pm}(x)=x_{ \pm} \mp \frac{1}{2} x_{\mathbf{h}}$.

For example, if $p_{0} \in \mathbf{n}_{+}$, then $U(\underline{\mathrm{t}})$ does not depend on $n_{+}(0)$. This failure of $\Omega_{p_{0}}$ to be injective for these $p_{0}$ is not very surprising, as the choice $p_{0} \in \mathbf{n}_{ \pm}$corresponds to taking starting values on one chiral branch of the $1+1$ dimensional light cone. Therefore it can be interpreted as an expression of the non-uniqueness of the Cauchy problem.

We will not be concerned with the question of injectivity of $\Omega_{p_{0}}$ for general $p_{0}$ and $G$ as before the loop group over a semisimple finite dimensional group $\mathbf{K}_{\mathbb{T}}$, but let us briefly indicate the heuristical arguments leading to the conclusion that for generic $p_{0}$ having non-zero parts in $\mathbf{n}_{+}$and $\mathbf{n}_{-}, \Omega_{p_{0}}$ is, at least formally, injective:

In the following we always choose a marking of the Lie algebra $\mathbf{k}_{\mathbb{q}}$ of $\mathbf{K}_{\mathbb{W}}$, i.e. besides of the Cartan subalgebra $\mathbf{h}_{\mathscr{C}}$ we also choose a basis $\Delta$ of simple roots, basis vectors $e_{\alpha}$ in every root space, constituting a Chevalley basis and nilpotent subalgebras $\mathbf{n}_{ \pm}$, such that the positive and negative roots are contained in $\mathbf{n}_{+}, \mathbf{n}_{-}$, respectively. We also define the Borel subalgebras $\mathbf{b}_{ \pm}=\mathbf{n}_{ \pm} \oplus \mathbf{h}_{\mathfrak{q}}$.

Using the grading of $\mathbf{g}$ given by $\operatorname{deg}\left(\lambda^{n} e_{\alpha}\right)=n+\mathrm{ht}(\alpha)$, one sees that the orbit is restricted to the set $\mathbf{m}_{0}$ of elements in $\mathbf{g} \cong \widetilde{\mathbf{g}}_{+}$of which the degree does not exceed the maximum or minimum degree of $p_{0}$. Therefore it will be finite dimensional, if $p_{0}$ contains only finite powers in $\lambda$. The function $U(\cdot)$ generically determines the projection of $\log g_{ \pm}$to $\mathbf{m}_{0}$, as was the case for the NLS.

Furthermore, because the second terms on the r.h.s. of the equations (23) and (24) vanish, if we project on $\mathbf{g} \ominus \mathbf{m}_{0}$, we get, also by similar arguments as for the NLS case, recursive equations for the higher powers of $\lambda^{-1}$ and $\lambda$ in 
$g_{-}$and $g_{+}$, respectively. These equations are formally solvable but in general very complicated, as they always contain infinitely many commutators with the degree 0 part of $\log g_{ \pm}$.

However, one has to be careful, whether the ansatz, being implicit in the expression $\log g_{ \pm}$is justified. The requirement that the exponential map is bijective on the small group $\mathbf{G}$ is hard to achieve in practice. It is not true even for the modified loop group definition given above. The injectivity of $\Omega_{p_{0}}$ is thus conserved only in an algebraic sense, obtained by considering the universal enveloping algebras rather than the Lie groups.

The class of algebraic geometric (finite gap) solutions $\left[g_{-}(\underline{\mathrm{t}})\right]$ of an integrable system is characterized by the condition that the tangent space of the flow at the starting point $\left[g_{-}\right]$is finite dimensional, i.e. there exist only finitely many linear independent $p_{j}$ such that all their flows are nontrivial. In the case of the NLS systems it suffices [1], section 4] to show that there exists one polynomial regular $\tilde{p} \in \mathbf{h}_{+}$such that its flow is trivial. Polynomial regularity for an element of $L^{a+} \mathbf{k}_{\mathbb{C}}$ shall mean that it can be written as a polynomial in the loop parameter $\lambda$ with leading coefficient being a regular element of the Lie algebra $\mathbf{k}_{\mathbb{C}}$. Further theorems concerning the existence of periodic algebraic geometric solutions are given in [1]. For a general account on algebraic geometric solutions see the article of Dubrovin, Krichever and Novikov [11].

\section{Group theoretic symmetry}

The above definition (2) of the flows makes the following definition of a symmetry quite natural:

Definition: A group theoretic symmetry for an integrable system of the kind described above is a differentiable automorphism $\phi$ of the group $\mathbf{G}$, which

a) respects the Birkhoff factorization, i.e. is also an automorphism of the subgroups $\mathbf{G}_{ \pm}$

b) its derivative $\mathrm{d} \phi$ leaves the Cartan subalgebra $\mathbf{h}$ and the generators $p_{0}, p_{1} \in \mathbf{h}$ of space and time translation invariant. 
In the case of an additional reduction, as for the $\mathrm{KdV}$ or $\mathrm{mKdV}$ systems, this should be supplemented by a consistency condition on $\phi$.

Invariance of $\mathbf{h}$ under $\phi$ is necessary to guarantee that $\phi$ reduces to a map on $\mathbf{H}_{-} \backslash \mathbf{G}_{-}$, which is the set of solutions of the system. The restriction (b) on $\phi$ can, and has to be loosened, in order to include space-time symmetries, i.e. symmetries which do not let invariant the flow parameters, for example the Poincaré symmetry, encountered in connection with the Leznov-Saveliev systems. We will formulate this generalization at the end of this section.

For a group theoretic symmetry $\phi$ we obviously have

$$
\tilde{g}(x, t)=\exp \left(-x p_{0}-t p_{1}\right) \tilde{g}_{-}=\tilde{g}_{-}(x, t) \tilde{g}_{+}(x, t),
$$

with $\tilde{g}=\phi(g), \tilde{g}_{ \pm}=\phi\left(g_{ \pm}\right)$. Therefore $\phi$ maps a solution of the system generated by the abstract data $\left(\mathbf{G}, \mathbf{G}_{-}, \mathbf{G}_{+}\right)$with starting point $g_{-}$to a solution of the same system with starting point $\tilde{g}_{-}$.

As the notions of a periodic and of an algebraic geometric solution can be expressed in a purely group theoretic way, it is easy to see that the classes of periodic and of algebraic geometric solutions are mapped to themselves. Every flow which acted trivial on the starting point $g_{-}$is mapped to a flow which acts trivial on $\tilde{g}_{-}$. This kind of symmetry will be used to model Bäcklund transformations for the generalized KdV equations.

If one has a Miura transformation mapping the solutions of an integrable system (1) to another integrable system (2), being invertible for a sufficiently large class of solutions of (2), and a symmetry of the second one, then it is possible to "pull back" this symmetry by the Miura transformation to a symmetry of the first one. This transformation we call a Bäcklund transformation.

Clearly, if the Miura transformation would constitute an isomorphism of Birkhoff factorizations, the Bäcklund transformations would not be of any interest, because then the symmetries of the systems could be easily translated into each other by purely group theoretic means. But for the generalized $\mathrm{KdV}$ equations and their counterpart, the generalized $\mathrm{mKdV}$ equations of Drinfel'd and Sokolov, this is not the case. There the Miura transformation is the projection of one coset space into another [4, 1], which can have a very complicated form if being expressed in coordinates of the Lie-Algebra $\mathbf{g}_{+}$. For the groups $\mathbf{G}=\mathcal{L S L}(n, \mathbb{C})$, calculating the inverse of the Miura transformation in general amounts to solving a system of $n-1$ differential equations of order $n-1$ and degree $n$. 
In the following we will elaborate on this standard example of a Bäcklund transformation.

The following reduction of the generalized NLS systems results in a generalization (not the one of Drinfel'd and Sokolov) of the modified KdV equations:

Let $\hat{\mathbf{g}} \subset \mathbf{g}$ be the covering Lie algebra of $\mathbf{k}_{\mathbb{T}}$ generated by the involution (notation as before):

$$
\sigma:\left\{\begin{array}{l}
\mathbf{h}_{\mathbb{C}} \ni x \mapsto-x, \\
e_{\alpha} \mapsto e_{-\alpha} \forall \alpha \in \Delta
\end{array}\right.
$$

So $\hat{\mathbf{g}}$ is generated by $\lambda^{2 n+1} \mathbf{h}_{\mathbb{T}}$, and the elements $\lambda^{2 n+1}\left(e_{\alpha}-e_{-\alpha}\right), \lambda^{2 n}\left(e_{\alpha}+e_{-\alpha}\right)$, $\alpha \in \Delta, n \in \mathbb{N}$.

The parameter-dependent modified $\mathrm{KdV}$ equation for $\mathbf{K}_{\mathbb{C}}=\mathrm{SL}(2, \mathbb{C})$,

$$
\psi_{t}=6\left(\psi^{2}+c\right) \psi_{x}-\psi_{x x x},
$$

$c$ being an arbitrary complex parameter, which was used by Miura [12] cannot be obtained by a group theoretic reduction of $\mathbf{g}$. It rather connects with a dynamical restriction of the flows to an affine subspace

$$
\mathbf{m}=\tilde{c} \sigma_{2}+\hat{\mathbf{g}}, \tilde{c}=\sqrt{-c}
$$

of $\mathbf{g}$. One may say that, by an additional symmetry of the third order flow of the NLS hierarchy, which yields the mKdV equation, a solution stays in $\mathbf{m}$, when its starting point $g_{-}(x, 0)$ was inside $\mathbf{m}$ for all $x$. Neither the existence of such a starting point, nor the above fact can be easily proved by group theoretic arguments.

However, we know that there exists a Miura transformation for every choice of the parameter $c$ connecting the corresponding $\mathrm{mKdV}$ with the $\mathrm{KdV}$ equation:

$$
u=\psi_{x}+\psi^{2}+c .
$$

We will now show, that there exists an analogous restriction of the generalized mKdV equations of Drinfel'd and Sokolov for higher groups $\mathbf{K}_{\mathbb{C}}=\operatorname{SL}(n, \mathbb{C})$ together with a group theoretic symmetry generalizing the construction for $\mathrm{SL}(2, \mathbb{C})$. Let us therefore recall the procedure of [四], which gives the generalized Miura transformations.

We restrict the group $\mathbf{G}$ to the subgroup $\check{\mathbf{G}}$ of loops in $L^{a} \mathbf{K}_{\mathbb{C}}$ which factorize through the map $\lambda \mapsto \lambda^{n}$ of $\mathbf{G}$ onto itself, where $n-1$ is the height 
of the unique highest root $\delta$, w.r.t. the chosen basis of simple roots. Let $\check{\mathbf{g}} \subset \mathbf{g}$ be the Lie algebra of $\check{\mathbf{G}}$, set $z=\lambda^{n}$ and choose the splitting

$$
\begin{aligned}
& \check{\mathbf{g}}_{-}=\left(\mathbf{g}_{-} \oplus \mathbf{n}_{-}\right) \cap \check{\mathbf{g}}, \\
& \check{\mathbf{g}}_{+}=\left(z \mathbf{g}_{+} \oplus \mathbf{b}_{+}\right) \cap \check{\mathbf{g}}
\end{aligned}
$$

of $\check{\mathrm{g}}$ and the momentum generator

$$
q_{0}=\sum_{\alpha \in \Delta} e_{\alpha}+z e_{-\delta} \in \check{\mathbf{g}}_{+},
$$

i.e. the subalgebra $\check{\mathrm{g}}_{-}$is enlarged, compared to the Birkhoff factorization of the NLS. By the procedure described in the section before, one gets an injective map $\Omega_{q_{0}}: \check{\mathbf{G}}_{-}\left(q_{o}\right) \backslash \check{\mathbf{G}}_{-} \rightarrow q_{0}+\mathcal{K}_{0}\left(\mathbb{R}, \mathbf{h}_{\mathbb{C}}\right)$.

Let $\mathbf{u} \oplus\left[q_{0}, \mathbf{n}_{-}\right]$be a vector space decomposition of the Borel subalgebra $\mathbf{b}_{-}$. In the case of $\mathbf{K}=\mathbf{s l}(n, \mathbb{C}), \mathbf{u}$ is the set of $\operatorname{sl}(n, \mathbb{C})$ matrices having non-zero entries only on the lowest row.

We now view $\mathcal{K}_{0}\left(\mathbb{R}, \mathbf{h}_{\mathbb{C}}\right)$ as a subspace of $\mathcal{K}_{0}\left(\mathbb{R}, \mathbf{b}_{-}\right)$. The set of functions $\mathcal{K}_{0}\left(\mathbb{R}, \mathbf{N}_{-}\right), \mathbf{N}_{-}$the group corresponding to $\mathbf{n}_{-}$, acts as a right transformation group on the latter by

$$
v^{\prime}(x)=\operatorname{Ad}\left(n_{-}^{-1}(x)\right) v(x)+n_{-}^{-1}(x) \frac{\mathrm{d} n_{-}(x)}{\mathrm{d} x}, n_{-}(\cdot) \in \mathcal{K}_{0}\left(\mathbb{R}, \mathbf{N}_{-}\right),
$$

$v(\cdot) \in \mathcal{K}_{0}\left(\mathbb{R}, \mathbf{b}_{-}\right)$. In $[\mathbb{1}]$ it was shown that by this action, $\Omega_{q_{0}}$ projects down to an injective map

$$
\Phi_{q_{0}}: \check{O}_{q_{0}}=\check{\mathbf{G}}_{-}\left(q_{0}\right) \backslash \check{\mathbf{G}}_{-} / N_{-} \rightarrow q_{0}+\mathcal{K}(\mathbb{R}, \mathbf{u}) .
$$

This map plays the rôle of the inverse scattering problem of the KdV equation. The projection

$$
\check{\mathbf{G}}_{-}\left(q_{0}\right) \backslash \check{\mathbf{G}}_{-} \rightarrow \check{O}_{q_{0}}
$$

is the generalized Miura transformation of Drinfel'd and Sokolov.

The triple $\left(\check{\mathbf{g}}, \check{\mathbf{g}}_{-}, \check{\mathbf{g}}_{+}\right)$is a reduction (in the sense of [1]) of the Manin triple $\left(L^{a} \mathbf{k}_{\mathbb{T}},{ }^{-} L^{a} \mathbf{k}_{\mathbb{T}},{ }^{+} L^{a} \mathbf{k}_{\mathbb{C}}\right)$, where ${ }^{-} L^{a} \mathbf{k}_{\mathbb{C}}$ is generated by the Cartan subalgebra of $\mathbf{k}_{\mathbb{T}}$ and all elements $\lambda^{k} e_{\alpha}$ with $h t(\alpha)+k>0$, and ${ }^{-} L^{a} \mathbf{k}_{\mathfrak{T}}$ is generated by all such elements with ht $(\alpha)+k<0$. The latter may be transformed first by an automorphism

$$
\begin{aligned}
\lambda^{k} e_{\alpha} & \mapsto \lambda^{\mathrm{ht}(\alpha)+k} e_{\alpha}, \\
\mathbf{h}_{\mathbb{C}} \ni x & \mapsto x
\end{aligned}
$$


then by the inner automorphism of $\mathbf{k}_{\mathbb{C}}$ conjugating $q_{0}$ into the Cartan subalgebra $\mathbf{h}_{\mathbb{C}}$. This is a transformation of the above Manin triple to the triple of the NLS equation, mapping the momentum generator of the $\mathrm{mKdV}$ system $q_{0}$ to the momentum generator $p_{0}$ of a system of NLS type. Thus the generalized mKdV systems of Drinfel'd and Sokolov are also embedded in the NLS hierarchy.

The above construction relating solutions of the mKdV and KdV systems may be varied for $\mathbf{K}_{\mathbb{C}}=\operatorname{SL}(n, \mathbb{C})$ in the following way:

Instead of identifying the spectral parameters of the $\mathrm{KdV}$ and $\mathrm{mKdV}$ model as above, we set

$$
z=\tilde{z}-c^{n}, c \in \mathbb{C},
$$

where $z$ and $\tilde{z}$ are the spectral parameters of the KdV and $\mathrm{mKdV}$ equations, respectively.

Let now $a_{j}, j=0, \ldots, n-1$ be the roots of the equation $\lambda^{n}=c^{n}$,

$$
a_{j}=c e^{\frac{2 \pi i(n-j)}{n}} \text {. }
$$

This gives

$$
\prod_{j=0}^{n-1}\left(\lambda-a_{j}\right)=\lambda^{n}-c^{n} .
$$

We choose the standard basis of simple roots $\left\{\alpha_{1}, \ldots, \alpha_{n-1}\right\}$ of $\mathbf{s l}(n, \mathbb{C}), \alpha_{j}=$ $e_{j j}-e_{j+1, j+1}, e_{j k}$ being the matrix units $\left(e_{j k}\right)_{l m}=\delta_{j l} \delta_{k m}$.

Setting $\tilde{z}=\lambda^{n}$ and performing the automorphism

$$
\begin{aligned}
e_{\alpha_{j}} & \mapsto\left(\lambda-a_{j}\right) e_{\alpha_{j}}, \\
e_{-\alpha_{j}} & \mapsto\left(\lambda-a_{j}\right)^{-1} e_{-\alpha_{j}}, \\
\mathbf{h}_{\mathbb{C}} \ni x & \mapsto x
\end{aligned}
$$

out of $q_{0}$ we obtain the element

$$
\tilde{q}_{0}=\sum_{j}\left(\lambda-a_{j}\right) e_{\alpha_{j}}+\left(\lambda-a_{0}\right) e_{-\delta} \stackrel{\text { def }}{=} \lambda A+\widetilde{C} .
$$

The diagonalization matrix of $A$ is $(Q)_{j k}=\sqrt{\frac{1}{n}} s^{j k}, s=e^{\frac{2 \pi i}{n}}$, all matrix indices now running from 0 to $n-1$. Let $B$ be a matrix which has entries only on one diagonal, i.e.

$$
(B)_{j k}= \begin{cases}b_{j} & \text { if } k=j+\epsilon \text { or } k+n=j+\epsilon \\ 0 & \text { otherwise }\end{cases}
$$


for some integer constant $0 \leq \epsilon \leq n-1$. The conjugation with $Q$ maps $B$ to

$$
\left(Q^{-1} B Q\right)_{j k}=s^{k \epsilon} \sum_{m=0}^{n-1} b_{m} s^{m(k-j)},
$$

The matrix $\widetilde{C}$ therefore changes to

$$
C=-c\left(\sum_{j=1}^{n-1} s^{j-1} e_{\alpha_{j}}+s^{n-1} e_{-\delta}\right),
$$

i.e. the matrix $\tilde{q}_{0}$ takes the form

$$
p_{0}+C=\sum_{j=0}^{n-1} s^{j} e_{j j}+C,
$$

where $p_{0}$ is the $x$-flow generator of the NLS systems.

The diagonal matrices, containing the fields, are transformed into offdiagonal matrices. This means that the above chain of transformations leads again to an element of $\Omega_{p_{0}}$ but now not contained in the section $\Omega_{p_{0}} \cap \hat{\mathrm{g}}$. It also maps $\check{\mathbf{g}}_{-}$and $\check{\mathbf{g}}_{+}$to the Birkhoff factorization $\mathbf{g}_{-}$and $\mathbf{g}_{+}$of the NLS models.

In analogy to the case $\mathbf{K}_{\mathbb{C}}=\operatorname{SL}(2, \mathbb{C})$ we now restrict the flows of the generalized NLS systems to the image of the generalized KdV systems under the above constructed Miura transformation depending on the parameter $c$. The matrix $C$ is therefore a generalization of the matrix $\tilde{c} \sigma_{2}$ in $(28)$.

As mentioned before, a Bäcklund transformation is modeled out of a Miura transformation between two integrable systems and a symmetry. We are therefore looking for an automorphism leaving $p_{0}+C$ and the image of $\mathbf{h}_{\mathbb{C}}$, in which the fields are contained, invariant. In addition it has to respect the Birkhoff factorization of the generalized NLS equations.

An obvious possibility is the following linear transformation of Chevalley generators:

$$
\begin{aligned}
e_{\alpha_{j}} & \mapsto s e_{\alpha_{j+1}}, j=1, \ldots, n-2, \\
e_{-\alpha_{j}} & \mapsto e_{-\alpha_{j+1}}, j=1, \ldots, n-2, \\
e_{\alpha_{n-1}} & \mapsto s e_{\alpha_{-\delta}}, \\
e_{-\alpha_{n-1}} & \mapsto e_{\alpha_{\delta}}
\end{aligned}
$$


and the resulting transformation of the Cartan subalgebra. We will not write down the general transformation of the fields explicitly.

In the case $n=2$ this all reduces to the well known procedure for the $\mathrm{KdV}$ system. It also reproduces the parameter-dependent Miura transformation, which can be calculated explicitly.

In the case $n=3$ we obtain the following explicit formulas $\left(s=e^{\frac{2 \pi i}{3}}\right)$ :

$$
\begin{gathered}
q_{0}=\left(\begin{array}{ccc}
0 & 1 & 0 \\
0 & 0 & 1 \\
\tilde{z} & 0 & 0
\end{array}\right), \\
\tilde{q}_{0}=\left(\begin{array}{ccc}
0 & \lambda-c s^{2} & 0 \\
0 & 0 & \lambda-c s \\
\lambda-c & 0 & 0
\end{array}\right), \\
p_{0}+C=\lambda\left(\begin{array}{ccc}
1 & 0 & 0 \\
0 & s & 0 \\
0 & 0 & s^{2}
\end{array}\right)-\left(\begin{array}{ccc}
0 & c & 0 \\
0 & 0 & c s \\
c s^{2} & 0 & 0
\end{array}\right) .
\end{gathered}
$$

The Miura transformation becomes more complicated, and is not explicitly solvable, since it is no more of Riccati type. We give it for $c=0$. If

$$
q_{0}+u=\left(\begin{array}{ccc}
0 & 1 & 0 \\
0 & 0 & 1 \\
u_{1}+z & u_{2} & 0
\end{array}\right)
$$

is an element of $\check{O}_{q_{0}}$ and

$$
U=\left(\begin{array}{ccc}
\psi & 1 & 0 \\
0 & \phi-\psi & 1 \\
z & 0 & -\phi
\end{array}\right)
$$

an element of $\check{\mathbf{G}}\left(q_{0}\right) \backslash \check{\mathbf{G}}$ which is mapped by the projection (35) to $q_{0}+u$, then the Miura transformation for the fields is

$$
u_{2}=(\phi+\psi)_{x}+\psi^{2}-\psi \phi+\phi^{2}
$$

and

$$
u_{1}=-u_{2} \psi+r_{x}+(\psi+\phi) r+\frac{1}{2} \psi \phi(\phi-\psi),
$$


where $r=\psi_{x}-\frac{1}{2} \psi(\phi-2 \psi)$. The symmetry transformation of the fields is

$$
\begin{aligned}
\psi & \mapsto \widetilde{\psi}=s^{2} \phi, \\
\phi & \mapsto \widetilde{\phi}=-s^{-1} \psi-s \phi .
\end{aligned}
$$

It is not clear, that the resulting Bäcklund transformation maps algebraic geometric into algebraic geometric solutions. Periodicity is not conserved in general. This has to be proven in every case separately by looking at the resulting differential equations. For the $\mathrm{KdV}$ equation with periodic boundary conditions, the Bäcklund transformation just amounts to adding one more gap to the spectrum of Hill's equation, or equivalently one more singularity to the Riemannian surface of an algebraic geometric solution [四]. A Bäcklund transformation for the group theoretic systems should therefore conserve the quality of algebraic geometricity.

The class of time-flow generators $q_{1}$, which are mapped by the above parameter-dependent transformations to elements commuting with $p_{0}$ is restricted. An example for such a generator is

$$
\tilde{q}=\left(z+c^{n}\right) q_{0}
$$

It is mapped to $\lambda^{n} p_{0}$. This means that we must investigate the mKdV equation for $q_{0}, q_{1}=z q_{0}$ with the transformed space-variable $\hat{x}=x+c^{n} t$ :

$$
\exp -\left(x q_{0}+t \tilde{q}\right)=\exp -\left(\left(x+c^{n} t\right) q_{0}+t q_{1}\right) .
$$

Thus a stationary solution for $c=0$ will be a wave propagating with velocity $-c^{n}$ for general $c$.

The symmetry automorphism (46) together with the generalized parameterdependent Miura transformation yields a Bäcklund transformation for the group theoretically generalized $\mathrm{KdV}$ systems in the case of $\mathbf{k}_{\mathbb{C}}=\mathbf{s l}(n, \mathbb{C})$. For other simple Lie algebras a similar construction should be possible by taking matrix representations which are reductions of the defining representation of an $\mathbf{s l}(n, \mathbb{C})$.

To show that the above definition of a symmetry is also of independent value, we provide at last the promised formulation of space-time symmetries of group theoretically describable systems.

If one chooses two generators $p_{0}, p_{1}$ for space and time flow, respectively, 
then in order to maintain the ZS type equation (6) for $V_{0}$ and $V_{1}$, it is enough to leave the scalar product $\langle\underline{\mathrm{t}}, \underline{\mathrm{p}}\rangle=x p_{0}+t p_{1}+\ldots$ in (2) invariant. When transforming $p_{0}$ and $p_{1}$ nontrivially, we must therefore also change the parametrization of space-time. The picture is similar to the "passive" one in special relativity.

An example for such a symmetry is provided by the implementation of the Lorentz group action in the group theoretic scheme for the Leznov-Saveliev systems. As we are working in light-cone coordinates, we write $\xi=t_{0}$ and $\eta=t_{1}$. There we have the following automorphisms:

The Cartan involution $T$ of the loop group,

$$
e_{\alpha} \rightarrow-e_{-\alpha}, \lambda \rightarrow \lambda^{-1}, \mathbf{h} \rightarrow-\mathbf{h}
$$

maps $p_{0}$ for the LS systems to $-p_{1}$ and vice versa. $T$ therefore amounts to the time reversal $\xi \leftrightarrow-\eta$.

The additional multiplication by -1 gives a space reversal $\xi \leftrightarrow \eta$. The boosts $\xi \mapsto \kappa \xi, \eta \mapsto \kappa^{-1} \eta$ are implemented by the automorphism

$$
\begin{aligned}
e_{\alpha} & \mapsto \kappa e_{\alpha}, \alpha \in \Delta^{+}, \\
e_{-\alpha} & \mapsto \kappa^{-1} e_{-\alpha}, \alpha \in \Delta^{+}, \\
\lambda & \mapsto \kappa^{n} \lambda,
\end{aligned}
$$

which is the identity on $\mathbf{h}_{\mathbb{C}}$.

The author thanks M. Schmidt and R. Schrader for helpful discussions on the subject. This work was supported by Deutsche Forschungsgemeinschaft as part of Sonderforschungsbereich 288 "Differentialgeometrie und Quantenphysik".

\section{References}

[1] G. Haak, M. Schmidt, R. Schrader, "Group theoretic formulation of the Segal-Wilson approach to integrable systems with applications", to appear in Rev. Math. Phys.

[2] R. Miura, ed., "Bäcklund Transformations", Lect. Notes in Math. 515, Springer-Verlag, New York, 1976. 
[3] V. V. Sokolov, A. B. Shabat, "(L,A)-Pairs and a Riccati type substitution", Func. Anal. Appl. 14, 148-140 (1980).

[4] V. G. Drinfel'd, V. V. Sokolov, "Equations of Korteweg-deVries type and simple Lie algebras", Soviet Math. Dokl. 23 457-462 (1981).

[5] A. N. Pressley, G. B. Segal, "Loop groups and their representations", Oxford University Press, 1985.

[6] V. G. Drinfel'd, "Quantum Groups", Proc. of the ICM-86, Berkeley, 798-820 (1986).

[7] I. B. Frenkel, "Orbital theory for affine Lie algebras", Invent. Math 77, 301-352 (1984).

[8] A. P. Fordy, P. P. Kulish, "Nonlinear Schrödinger equations and simple Lie algebras", Comm. Math. Phys. 89, 427-443 (1983).

[9] A. N. Leznov, "On the complete integrability of a nonlinear system of partial differential equations in two-dimensional space", Theor. and Math. Phys 42, 225-229 (1980).

[10] H. Wu, "Non-linear partial differential equations via vector fields on homogenous Banach manifolds", to appear in Ann. Glob. Ana. Geom.

[11] B. A. Dubrovin, I. M. Krichever, S. P. Novikov, "Integrable Systems. I", in: V. I. Arnol'd, S. P. Novikov (eds.), Dynamical Systems IV, Springer 1990.

[12] R. M. Miura, "Korteweg-de Vries equation and generalizations. I. A remarkable explicit nonlinear transformation", J. Math. Phys. 9, 1202-1204 (1968). 